Article

\title{
Fractal, Scale Free Electromagnetic Resonance of a Single Brain Extracted Microtubule Nanowire, a Single Tubulin Protein and a Single Neuron
}

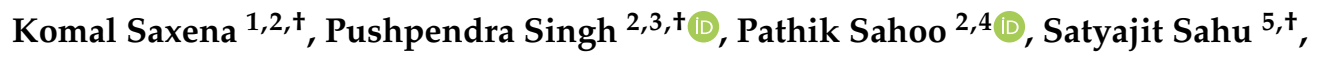 \\ Subrata Ghosh ${ }^{4,+}$, Kanad Ray ${ }^{3}$, Daisuke Fujita ${ }^{2}$ and Anirban Bandyopadhyay ${ }^{2, *(D)}$ \\ 1 Microwave physics laboratory, Department of Physics and computer science, Dayalbag Educational Institute, \\ Agra, Uttar Pradesh 282005, India; komal03oct@gmail.com \\ 2 Advanced Key Technologies Division, National Institute for Materials Science, 1-2-1 Sengen, Tsukuba, \\ Ibaraki-3050047, Japan; singhpushpendra548@gmail.com (P.S.); 2c.pathik@gmail.com (P.S.); \\ FUJITA.Daisuke@nims.go.jp (D.F.) \\ 3 Amity School of Applied Science, Amity University Rajasthan, Kant Kalwar, NH-11C, Jaipur Delhi Highway, \\ Jaipur, Rajasthan 303007, India; kanadray00@gmail.com \\ 4 North Eastern Institute for Science and Technology, NEIST, Jorhat, Assam 785006, India; ocsgin@gmail.com \\ 5 Department of Physics, Indian Institute of Technology, Jodhpur, Rajasthan 342037, India; satyajit@iitj.ac.in \\ * Correspondence: anirban.bandyo@gmail.com \\ + These four authors contributed equally to this manuscript.
}

Received: 1 March 2020; Accepted: 2 April 2020; Published: 6 April 2020

check for updates

\begin{abstract}
Biomaterials are primarily insulators. For nearly a century, electromagnetic resonance and antenna-receiver properties have been measured and extensively theoretically modeled. The dielectric constituents of biomaterials-if arranged in distinct symmetries, then each vibrational symmetry-would lead to a distinct resonance frequency. While the literature is rich with data on the dielectric resonance of proteins, scale-free relationships of vibrational modes are scarce. Here, we report a self-similar triplet of triplet resonance frequency pattern for the four- $4 \mathrm{~nm}$-wide tubulin protein, for the 25-nm-wide microtubule nanowire and 1- $\mu \mathrm{m}$-wide axon initial segment of a neuron. Thus, preserving the symmetry of vibrations was a fundamental integration feature of the three materials. There was no self-similarity in the physical appearance: the size varied by $10^{6}$ orders, yet, when they vibrated, the ratios of the frequencies changed in such a way that each of the three resonance frequency bands held three more bands inside (triplet of triplet). This suggests that instead of symmetry, self-similarity lies in the principles of symmetry-breaking. This is why three elements, a protein, it's complex and neuron resonated in $10^{6}$ orders of different time domains, yet their vibrational frequencies grouped similarly. Our work supports already-existing hypotheses for the scale-free information integration in the brain from molecular scale to the cognition.
\end{abstract}

Keywords: microtubule; neuron; electromagnetic resonance; antenna; receiver; protein; fractal; impedance spectroscopy; transmission spectrum; reflectance spectrum

\section{Introduction}

A significant portion of our brain exhibits a scale-free dynamic in the electrical field potentials and also in the magnetic resonance responses [1]. Temporal correlations of brain's cognitive responses are widely studied [2], however, no reports exist for the scale-free temporal correlations connecting a single protein to a single neuron via intermediate structures. There are theoretical studies which claim scale-free vibrational link among 12 different time domains in the brain [3], extending the initial triplet of triplet fractal resonance chain proposal of Ghosh et al. [4]. Fractal-antenna-like features in 
the biomaterials have widely studied the topic of interest in recent times [5], Also, fractal geometry has been useful to design small and multiband antennas for wireless applications [6]. Establishing a connection is important since Ghosh et al. [7] have shown using coaxial probe that thus far believed micro and neurofilaments are not silent inside the axon initial segment, AIS, they vibrate and exchange signal with various dendritic and axonal branches before the soma builds up the axon potential [7]. Dielectric resonance measurement is carried out in two ways, either by measuring the reflectance and transmittance spectra or by measuring the impedance of proteins. These studies started in the 1930s and finding the fractal feature in the resonance frequency spectra is an abundant tool, frequently used in wide ranges of research fields. Therefore, be it a single tubulin protein, or a single microtubule or a neuron membrane, the dielectric resonance measurement is a routine task except that we use precise coaxial probe which because of its atomic sharp needle ensures that the precise contact is made with the material and environmental noise is drastically reduced. Impedance spectroscopy of microtubules was carried out in isolated conditions and also when these filaments are located in the neuron in a seminal work [8]. Extending the work further, Agrawal et al. [9] used a scanning dielectric microscope to image how ions move (milliseconds) live; as well as how the dipoles of filaments in a living cell of a neuron resonate (microseconds) in two distinct time domains to build a temporally correlated energy exchange to decide whether to fire or not [9]. Microtubules have been predicted as circular waveguides and resonators [10], and as an information processing device in the brain [11], which was confirmed by Sahu et al. in 2013 [12].

On the other hand, results from the electromagnetic resonance studies on tubulin protein and microtubules have been already theoretically predicted by the gold standard RRM model [13] and experimentally verified by Sahu et al. [14]. Regarding measurements of the dielectric resonance of neuron membranes, the literature is rich in advocating the fact that neuron membranes have wide ranges of distinct resonance frequencies, be it myelinated or not, it does not matter. The electromagnetic signal is not a movement of mass, extracellular materials, myelin sheath or fat coating cannot stop it, at wide ranges of high frequencies they do not resonate. Therefore, on the background of well documented and understood electromagnetic resonance of proteins and biomaterials, we investigate here the pure resonance frequencies of these materials.

In most of the dielectric measurements of proteins or biomaterials, the material is dissolved into the solution and the electrodes are dipped to make a contact with many elements since the background response of solution and biomaterial + solution are identical, the minimum differences are subtracted and the resultant signal is claimed as the pure material property. Consequently, the researchers derive non-physical results e.g., a metal-like ampere level current flow in tubulin $(1 \mathrm{~A}$ current in tubulin solution at $0.2 \mathrm{~V}, 0.2 \Omega$ resistance, like a superconductor, $15 \mu \mathrm{A}$ at $1 \mathrm{~V}$ for microtubule solution [15] and a massive microfarad capacitance in microtubule [16], quantum-like near-ballistic resistance without any electromagnetic resonance $(97.4 \mathrm{k} \Omega$ ). Collisionless quantum transport of electrons under massive noise in water along the microtubule surface at ambient atmosphere is non-physical. Moreover, metal-like current flow and an extreme insulator-like capacitance cannot be together: it should be either metal or an insulator if the milliampere-order current does not burn it. The literature is rich in non-physical claims, e.g., microtubules transport ions through their surface like metals-even when surrounded by water [17]. When a neuron membrane cannot transport ions through its surface, it transmits ions vertically through ion channels and an electric field horizontally along the membrane length. Apparently, to transmit vesicles, microtubules have devised massive walking mechanisms for the molecular motors. It is difficult to establish logically how one C-terminus captures ions and gives it to another C-terminus-and the efficiency for this is comparable to that of a metallic transmission. Researchers claim that in deionized water $(18.2 \mathrm{M} \Omega$ ) — which itself transmits microampere level currents at $1 \mathrm{~V}$ - they are able to measure biomaterials that send nanoampere or picoampere-level currents.

Tubulins self-assemble rapidly under electromagnetic pumping, though previous report existed, it was shown with better clarity by Sahu et al. [18], however Sahu et al. [18] have carried out impedance or AC resistance measurement of microtubule shown in Figure 1a by AC blocking and DC blocking, 
manually creating neutralization circuits for all 400 points measurements in the frequency spectrum of $\mathrm{kHz}, \mathrm{MHz}$ and $\mathrm{GHz}$ range. One such example circuit is shown in Figure 1b,c. Nearly 400 distinct circuits were created, no impedance analyzer was used to give a blind run because if there is a nonlinear property in the frequency for any material, no machine is designed to neutralize effects over such a long frequency range. Sahu et al. [18]'s painstaking measurements were difficult to follow because each data point requires a separate optimization circuit construction. Dipping biomaterials in solution and then measuring the transmission from one end of the beaker to another replicates the water response (background data-sample data, even nonlinear features look similar with and without water). The biomaterials are simply impure in the large number of published reports, buffer salts are always there $[8,19,20]$. Such claims arise because the necessity of connecting one molecular unit, e.g., a microtubule or one protein molecule with the electrode is supremely important for any device or material characterization, this fundamental principle of material characterization is ignored in almost every single study performed other than Sahu et al. [18]. The basic principle of normalization of electrode effect arises only when there is a proper contact, most reports are carried out in a non-contact or poor contact scenario, whereas AFM (Atomic Force Microscoppy)- or STM (Scanning tunneling Microscopy)-connected piezo-motor-controlled tips are the only reliable way to do it. The non-availability of such setups may have led to the generation of plethora of non-physical results, most of them suggest, microtubules are both superconductors and supercapacitors at the same time. Moreover, the impurity of water that itself electromagnetically resonates in the $\mathrm{kHz}$ (ions in water resonates in the entire $\mathrm{kHz}$ range) and in the $10-80 \mathrm{GHz}$ domain masks the biomaterials property. The problem is genuine and critical. Sahu et al. [18], and later Ghosh et al. [7], addressed this concern in their work, following two distinct routes.

For years, Sahu et al. [18] carefully mapped how long biomaterial needs to be dried so that it does not degrade, but electrode-solvent-electrode transport is neutralized. During optimization, the deformity in the quantum tunneling images or atomic force microscopes, RAMAN-FTIR spectra (single spot on a single element) and current-voltage characteristics were carefully optimized. While draining the excess water, setting the proper drying conditions and flushing the dry $\mathrm{Ar}^{+}$gas around its measuring environment was optimized for varying times so that the ionic responses of the biomaterials (hysteresis in current-voltage characteristics, noise in RAMAN-FTIR peaks, etc) were at a minimum. Note that no one could isolate internal water totally from a biomaterial without destroying it. We need to measure ionic transport, but that transport should be contributed by protein surface, filament surface or internal biomaterial structure, not by the solution. However, Ghosh et al. [7] found that simply drying is not enough: Ions and water layers need to be balanced with the structural stability of the biomaterials, or else the optimization fails. Instead, he used a coaxial probe Au-insulator-Pt to insert the atomic sharp measuring Pt probe into the sample while outer Au layer of the coaxial probe took the ionic signal to the ground, or measured by a current meter or impedance analyzer (Figure 1d,e). Note that 3-4 molecular layers of water coating were always present and triggered an ionic transport [21] but we got the $\mathrm{kHz}$ domain resonance due to the molecular surface. Therefore, two efforts were made: first, reducing the water layer so that only the surface ions flow between the electrodes, and second, using coaxial probe connected to a piezo motor enabling a controlled precise route to measure the ionic conduction of any biomaterial surface or inside. Another unique advantage of this probe was that one could measure the dipolar oscillations at $\mathrm{MHz}$ or $\mathrm{GHz}$ using the inner $\mathrm{Pt}$ probe simultaneously, with the ionic signal using Au electrodes as shown earlier by Ghosh et al. [7]. 

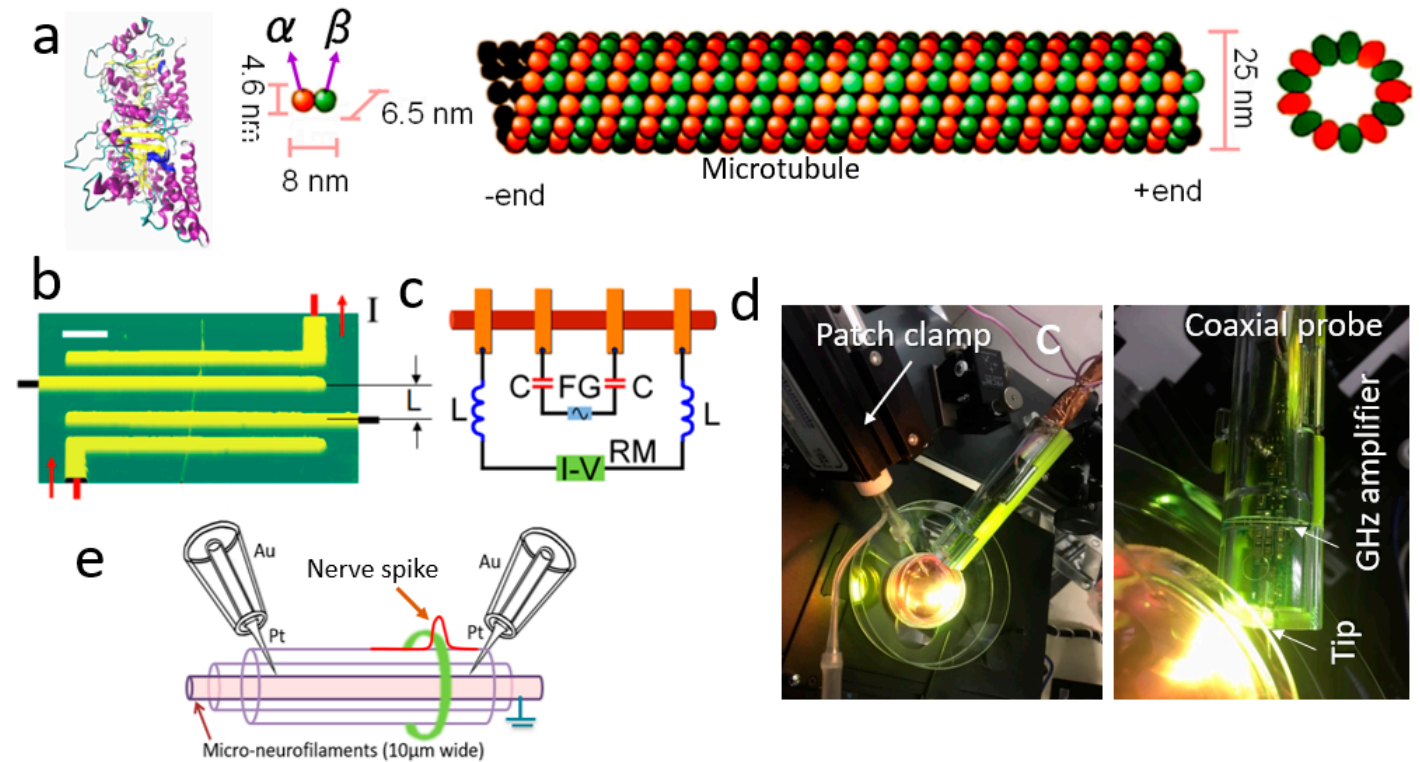

Figure 1. Experimental setups used for the background electromagnetic resonance measurement of microtubule and neuron; (a) A single tubulin protein structure is represented using a pair of balls; its dimensions are noted around it. Right, microtubule nanowire made of tubulin proteins; (b) SEM image of four probe single microtubule device, scale bar is $400 \mathrm{~nm}$; (c) Schematic presentation of the four-probe setup. $\mathrm{L}$ is an inductor to block AC current to the DC resistance measurement setup and $C$ is a capacitor to block the DC current; (d) Experimental setup for combined patch-clamp and our coaxial probe measurement on a cell culture plate. The glasstube of the patch-clamp was supplied with a buffer salt solution to maintain the pressure of the membrane-glass patch. The atomic resolution tip was connected to a $\mathrm{GHz}$ amplifier for instant signal amplification. Patch clamps sensed only ion flows that resonated at $\sim 1 \mathrm{~ms}$, read only one data and could not sense anything below 50-100 nm. In contrast, our recently invented coaxial atom probe (Figure 1d) sensed several time domains at a time, even below $0.5 \mathrm{~nm}$. Its cavity between a Pt metal needle and an Au metal cylinder traps (10-20 watt) vibrations in its vicinity, the cavity's conical geometry amplified that signal, and the embedded dielectric resonator (glass) increased $\mathrm{Q}$ factor $\left(\sim 10^{5}\right)$ at all frequencies that its conical geometry allowed ( $3 \mathrm{kHz}$ to $40 \mathrm{GHz}$ ). Unlike the patch-clamp, the atom probe read four signals at a time, filtered the noise, measured four distinct vibrations of protein complexes, noise-free $\left(\mathrm{S} / \mathrm{N} \sim 10^{5}\right)$, deep inside a neuron; (e) A pair of coaxial probes measured the passage of nerve spikes along the central filamentary region (right).

Since the 1930s, the practice of measuring biomaterials in hugely covered water, has been repeated continuously. The water layer around protein molecule is essential and its dielectric property of that water layer plays a vital role [22]. However, in the earlier times, 1930-1980, the researchers restricted themselves into the $\mathrm{GHz}$ or microwave domain where inions do not resonate, ions resonate in the $\mathrm{kHz}$ range, therefore protein data were not masked by ions moving between electrodes in the solution. However, there was another problem. Though water resonates $6-80 \mathrm{GHz}$ and demonstrates a nonlinear variation of dielectric constant, now if we dissolve proteins into the water and then carry out the measurement in GHz ranges, then, it would be water's nonlinear resonance behavior not that of proteins. However, the 4-nm protein nanoparticle's dielectric constant is measured around 2-3 $(\epsilon)$, whereas $\epsilon$ for similar nanoparticles would be around a few hundreds, around 10 at zero AC signal is a good theoretical prediction by Nanopoulos [23]. Since the background subtraction does not work when the water interacts with the biomaterial, it leads to non-physical results. Water molecules make an integral part of the protein molecule [24]. Buried water molecules hold proteins energy landscapes [25]. Sharp ripples in the normalized plots could be water interacting with the unwanted molecules in the buffer solution and delivering a response, unrelated to the protein. Consequently, 
the electromagnetic measurement in the $\mathrm{MHz}$ region is safe from water and ions, pure dipolar response is possible to capture, Sahu et al. [18] concentrated in that region.

For the survival of natural conformation and dynamics, biomaterials need water. If removed, the structure deforms, disintegrates. Since the pioneering work of Langmuir-who argued that a two to the three-molecular layer of water always remains as hydration shell around a biomolecule and that is essential for expressing the biomolecular property-we follow that in the experiment. We let the molecule dry over $2 \mathrm{~h}$ in the $70 \mathrm{~L}$ refrigerator, measured the vibration on the surface of the molecule using a piezo resonator connected to a quartz crystal micro-balance, FTIR-RAMAN and finished experiment rapidly - in the order of minutes. Between experiments, we added culture solution of neuron and or buffer solution for proteins and microtubules. One could also monitor current-voltage characteristic during drying, Ghosh et al. [7] created a prescription on how the feature changes to an optimized level. Once we are in the favorable wet domain, measurements of electromagnetic resonance are carried out.

\section{Experimental Section}

\subsection{Neuron Culture on Pre-grown Electrodes for Simultaneous Patch-Clamp and Wireless Axon Initial Segment (AIS) Experiment}

The sample, rat brain hippocampus (Hi); $\geq 0.25 \mathrm{~mL}$ embryonic neuron cell suspension (Lonza Inc.), was stored in the liquid nitrogen $\left(\mathrm{LN}_{2}\right)$ environment. We prepared the solution for a neuron culture following the procedure outlined by Lonza company manual. Transferred the cell suspension to the pre-grown Au electrode grids as shown in Figure 1d, randomly spaced electrodes were grown on a $\mathrm{Si} / \mathrm{SiO}_{2}$ substrate then coated with poly-D-lysine. The Au electrode surface was etched out for better electronic transmission. Incubate the cells for $4 \mathrm{~h}$ at $37^{\circ} \mathrm{C}$ in a $5 \% \mathrm{CO}_{2}$ incubator. Remove the medium from the cells, leaving a small volume to ensure the cells do not dry out. Add fresh, pre-warmed medium. Shift sections of the growing neurons using atom probe connected to high-resolution manipulators so that they grow, as we desire around the electrodes. Incubate the cells at $37^{\circ} \mathrm{C}$ with $5 \%$ $\mathrm{CO}_{2}$. After the initial medium change on day 5 , replace $50 \%$ of the growth media every 3 to 4 days. Warm an appropriate amount of medium to $37^{\circ} \mathrm{C}$ in a sterile container. Remove $50 \%$ of the medium from the cell culture. Replace with the warmed, fresh medium and return the cells to the incubator. Positional adjustment of neural branches around the electrodes of the grid continues until day 8-9.

We used 6-14-day-old rat hippocampus cultured neuron cells for the simultaneous patch-clamp and atom probe experiment. Note that atom probes are insulated except the extreme edge $\sim 2 \mathrm{~nm}$ to avoid electrical leakage from the cytoskeleton. Atom probes are scanning tunneling microscope tips coated with insulated resins. We carried out patch-clamp experiments $(\sim 150 \pm 12 \mathrm{~mm}$ wide, $n=35$, it means 35 neurons studied) for DC electric bias only. For measuring the neuron firing current, patch-clamp parameters were $\mathrm{R}_{\text {seal }} \sim 5 \pm 1 \mathrm{G} \Omega, n=35 ; \mathrm{R}_{\text {elec }} \sim 80 \pm 10 \mathrm{M} \Omega$, the success rate of proper clamping was $55 \%$ (For Figure 3, $n=35$, i.e., 35 pairs of neurons were measured in 35 devices). For a neuron, a surface electrode contact with the membrane shorts the electrical nerve impulse; however, a proper atomic electrode-axon core contact was essential for sensing a synchronous signal along the length of the neuron through the axon core. By measuring the resonance frequency, we confirm that we have touched the proper location inside the neuron, a composition of resonance frequencies was particular to a material. The resonance measurements are carried out using various impedance analyzers, vector analyzers and spectrum analyzers $[12,14,18,26]$. In Figure 3, the perpendicular electrodes do not touch, keeps $\sim 2-3 \mathrm{~nm}$ separation with the microtubule.

\subsection{Reconstitution of Microtubule}

Microtubules are extracted from porcine brain by Cytoskeleton (Denver, CO, USA), we purchased tubulin protein including all associated tubulin-to-microtubule conversion kits and reconstituted microtubule in our laboratory. Purified microtubule subunits (tubulins) were preserved at $-80^{\circ} \mathrm{C}$. 
To polymerize tubulin $[27,28]$, into $6.5-\mu \mathrm{m}$ long microtubules, $160 \mu \mathrm{L}$ of microtubule cushion buffer ( $60 \%$ v/v glycerol, $80 \mathrm{mM}$ PIPES $\mathrm{pH} 6.8,1 \mathrm{mM}$ EGTA, $1 \mathrm{mM} \mathrm{MgCl} 2)$ was added to $830 \mu \mathrm{L}$ of general tubulin buffer ( $80 \mathrm{mM}$ PIPES $\mathrm{pH}$ 7, $1 \mathrm{mM}$ EGTA (Ethylene glycol tetraacetic acid), $2 \mathrm{mM} \mathrm{MgCl}$ ) and $10 \mu \mathrm{L}$ of $100 \mathrm{mM}$ GTP (Guanosine 5'-triphosphate) solution. This mixture was kept in an ice bath for $10 \mathrm{~min}$. From this mixture, $200 \mu \mathrm{L}$ solution was added to $1 \mathrm{mg}$ of tubulin and again it was incubated in an ice bath for $10 \mathrm{~min}$. Afterward, this stock was placed in an incubator at $35-37^{\circ} \mathrm{C}$ for $40 \mathrm{~min}$. Now, to stabilize microtubules $20 \mu \mathrm{L}$ of Paclitaxal dissolved in anhydrous DMSO (Dimethyl sulfoxide; methyl sulfoxide) was added to the solution and it was incubated for a further $10 \mathrm{~min}$ at $37^{\circ} \mathrm{C}$. The microtubule length was tuned $\sim 4-20 \mu \mathrm{m}$. Actin microfilament was prepared following the guidelines from Cytoskeleton.

\subsection{Preparation of Protein Sample}

Proteins were kept at $-80^{\circ} \mathrm{C}$. Protein solutions were dropped on the nanogap electrode system under 4V DC electric field so that proteins are oriented. For STM studies, nanomolar solutions were dropped on a freshly cleaved HOPG (Highly oriented pyrolytic graphite) substrate. Proteins and its complexes are scanned only under resonant AC frequencies (all resonance frequencies are noted in the Figure 2 plot); else, they break apart. The nanomolar tubulin protein solution was dropped on a four-probe Au electrode junction when the electric field of $0.5 \mathrm{~V}$ and $0.5 \mathrm{~V}$ was $\mathrm{ON}$ across electrodes EF and GH (see Figure 3c), respectively and independently.
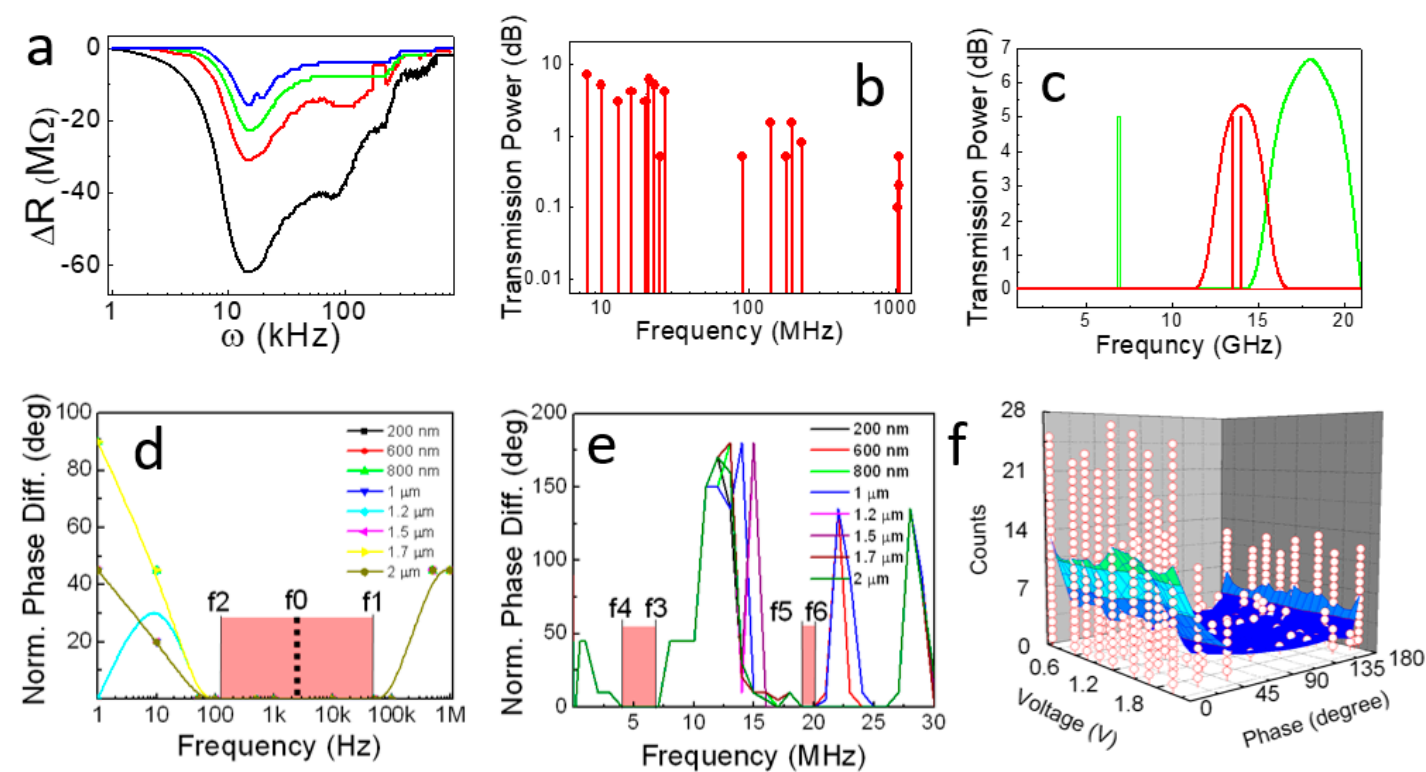

Figure 2. Fractal electromagnetic resonance band of a single brain extracted microtubule; (a) Difference in DC resistance (normalized) with AC pumping, (1 $\mu \mathrm{Hz}$ to $1 \mathrm{MHz} \mathrm{AC}, 5$ (black), 4, 3, 2, 1 (blue) $\mathrm{V}_{\text {rms }}$, important part is zoomed), it was measured using Figure 2d circuit; (b) Transmission output in dB across the microtubule device for $25 \mathrm{~dB}$ input; (c) Output transmission in $\mathrm{dB}$ appeared as a Gaussian distribution, sharp rectangular pulses depict the input signals (green $\sim 7 \mathrm{GHz}$, red $\sim 13 \mathrm{GHz}$ ). Normalization was

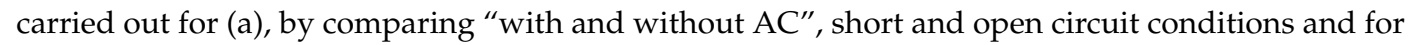
(b) and (c) short and various open-circuit conditions, like air-to-air, along silicon substrate, air-to-silicon, air-to-electrode and silicon-to-electrode; $(\mathbf{d}-\mathbf{f})$ phase difference between input and output AC signal as a function of frequency. All data were normalized considering open and short circuit conditions (device length varied between $200 \mathrm{~nm}$ to $2 \mu \mathrm{m}$ ). Here f1-f2, f3-f4 and f5-f6 are three bands where input-output phase difference was zero; (f) Between $10 \mathrm{kHz}$ and $50 \mathrm{MHz}$, statistical counts were made for the occurrence of particular phase, irrespective of applied AC frequency, which showed that only the phase differences as multiples of $45^{\circ}$ were observed. 


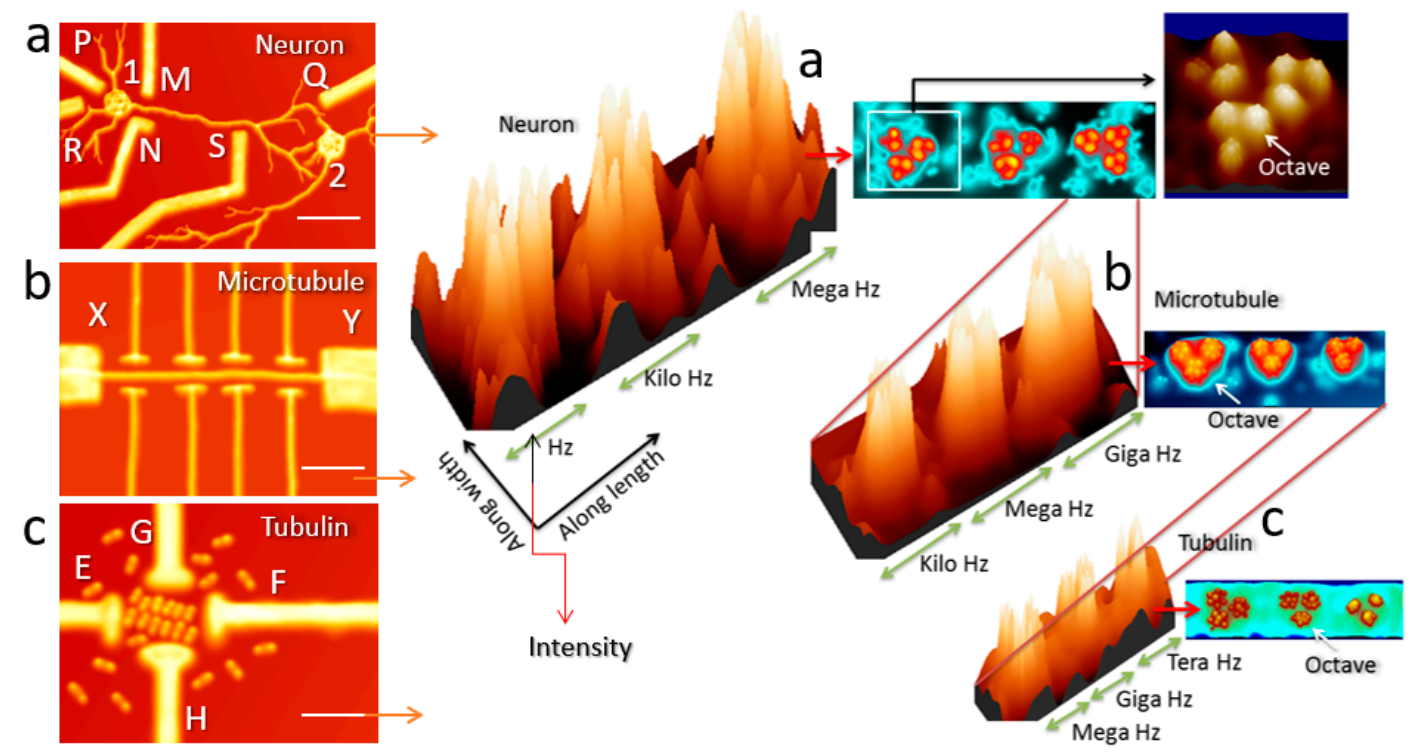

Figure 3. Self-similar resonance bands for neuron, microtubule and tubulin; (a) Microscope image Au electrode grid, rat hippocampal neuron, scale bar $=10 \mu \mathrm{m}$. PQ and RS are non-contact electrodes to supply horizontal AC signal. MN electrodes are just across the AIS; (b) Atomic force microscopy (AFM) of a single microtubule, scale bar $=120 \mathrm{~nm}, \mathrm{XY}$ are horizontal electrodes, the remaining four are transverse electrodes; (c) AFM of a tubulin substrate, scale bar $=50 \mathrm{~nm}$. The three log-log scale plots of resonance frequency corresponding to the three experimental setups of panels $a, b$ and $c$, are given to their right respectively. For panel A, the patch-clamp measured current difference between neuron 1 the soma and neuron 2 the soma is the vertical axis $\operatorname{Imax}=10 \mathrm{nA}, \operatorname{Imin}=1 \mathrm{pA}(\sim 80-2 \mathrm{mV})$. Two horizontal axes, one along the length ( $\mathrm{L}=50 \mathrm{~Hz}$ to $2.5 \mathrm{kHz}, 2.5$ to $250 \mathrm{kHz}$ and 1 to $30 \mathrm{MHz}$ ) and the other along the width $\mathrm{W}$. Wherein, $\mathrm{W}=\mathrm{L} \times 0.3$, if $\mathrm{L}=1 \mathrm{~Hz}$, then, $0.3 \mathrm{~Hz}$ to $1.3 \mathrm{~Hz}$ variation was made along the vertical axis. Thus, the vertical axis is linear frequency width, its upper and lower limits are percentage values $\mathrm{W}_{\min }=-30, \mathrm{~W}_{\max }=+30$ for all panels $\mathrm{a}, \mathrm{b}$ and $\mathrm{c}$. For panel $\mathrm{b}$ and $\mathrm{c}$, the $3 \mathrm{D}$ resonance plot's vertical axis is relative $\mathrm{AC}$ power transmission $\left(\mathrm{P}_{\text {output }} / \mathrm{P}_{\text {input }}=\mathrm{P}(0<\mathrm{P}<1)\right.$ ) across the horizontal $\mathrm{XY}$ and $\mathrm{EF}$ electrode pairs respectively. Horizontal axes are frequencies for all three panels. For panel (a) the length, width vertical ranges are noted above, for panel $b$ ranges are (triplet band) $10-300 \mathrm{kHz}, 10-230 \mathrm{MHz}$ and 1-20 GHz; for panel c ranges are (triplet band) $30-450 \mathrm{MHz}$, 1-250 GHz and 20-350 THz. To the right of all three 3D plots we show the corresponding vertical visuals, panel a is zoomed.

\section{Results and Discussion}

\subsection{Triplet of Triplet Fractal Electromagnetic Resonance Band of Single Brain Extracted Microtubule}

We provide more profound support to an earlier experimental observation by Pizzi et al. [29] that microtubule nanowire acts as both antenna and receiver during long-range communication. Microtubule was synthesized by the standard protocol described elsewhere [28]. We grow four $200 \mathrm{~nm}$ wide parallel gold electrodes on top of a single microtubule via e-beam lithography method (Figure 1b), both two probe and four probe measurements are carried out. In the initial days, we used to grow electrodes on the nanowire (11\% device survived), similar to carbon nanotube measurements, however, later we changed and dropped microtubule or biomaterials on the pre-grown electrodes. The method helps in the survival of a greater number of devices, though the formation of a proper contact became challenging ( $88 \%$ devices made good contact).

Figure 2 demonstrates a series of electromagnetic resonance measurements. Normally, after forming a biomaterial-electrode contact transmission and reflection coefficients are measured under AC signal. The peaks at specific resonance frequencies are considered to be the resonance frequencies. For years, Sahu et al. [18] and Ghosh et al. [7] adopted a different methodology, they observed at which 
frequencies the DC conductivity shows a sharp increase, it means, a dual check on resonance. To detect a resonance peak of a single microtubule nanowire, here, either we measure drop in DC resistance under AC pumping using the circuit in Figure $1 b$, (see Figure $2 a$ ) or, we measure transmission coefficients along the length of the biomaterial in $\mathrm{dB}$ under $\mathrm{AC}$ signal varying the frequency from $1 \mathrm{~Hz}$ to $20 \mathrm{GHz}$ (Figure $2 b, c)$ using a vector analyzer. Though subtracting background data are a common practice, we never do that, only for the reason that a blind subtraction brings noise artifacts as peaks and neglects important ones. In all resonance peak detection, we neglect open and short circuit responses to avoid noise. The peaks that appear in the open and short conditions are simply deleted; we consider that peak region as non-accessible. Finally, we observe three distinct regions. First, 10-100 kHz domain (Figure 2a) was depending on the AC bias amplitude, we observe the superposition of three distinct peaks of resistance loss, which becomes more distinct with more injection current, finally, we get quality factor, $\mathrm{Q} \sim 3-5$. Second, $8-240 \mathrm{MHz}$, (Figure 2b) peaks are sharp ( $\sim$ 100-300), energy is distributed nonlinearly among particularly allowed 8 resonance peaks in this domain; microtubule's length strictly determines which resonance frequencies are to be blocked. In more than $88 \%$ of devices, we found that the peaks with low intensities blink, i.e., appear and disappear, some peaks are negative, and some peaks are positive while continuously changing intensities. $\mathrm{MHz}$ peaks of microtubule are the treasure of enormous dynamic activities. Finally, in the 7-13 GHz domain, (Figure 2c) we have two Gaussian-like resonant transmissions if single microtubule was pumped at $7 \mathrm{GHz}$, near $19 \mathrm{GHz}$ we get a transmission band, which was remarkable since frequency was amplified. It means we get very different kinds of signal as output than what we send as input. At $13 \mathrm{GHz}$ pumping we observe another Gaussian-like stochastic resonance, however, in this case, it was peaked around $13 \mathrm{GHz}$ only.

The $\mathrm{kHz}$ and $\mathrm{GHz}$ bands do not change the phase of AC input signal (Figure 2d) while a quantized phase modulation by $\frac{n \pi}{4}$ occurs during MHz transmission across the microtubule (Figure 2e,f), which suggests that $\mathrm{MHz}$ band was strongly engaged in electromagnetic radiation. Quantization of phase naturally by microtubule to a non-coherent AC signal was a remarkable discovery for multiple reasons. First, it suggests that for phase-coherent signal transmission we do not need to send phase-coherent electronic, magnetic or electromagnetic signal through the microtubule, microtubule spontaneously converts non-coherent signals into a coherent one. However, depending on the input frequency, the output signal gets a predetermined phase.

Using CST (computer simulation technology), we created several model structures of tubulin, microtubule and single neuron and solved Maxwell's equations using the time domain solver. Two ports were connected to the two ends of the microtubule, tubulin and neuron and we could estimate electromagnetic energy absorption and radiation behaviors. Helical structures have unique properties. The positive and negative resonance at different frequencies ensure that-for a particular resonance frequency - if a microtubule's one end acts as a receiver, the other end would act as an antenna, the selectivity of signal frequency was independent of its length. However, radiation resistance was $R=80 \pi^{2}\left(\lambda_{p} / \lambda\right)^{2}$, where $\lambda_{p} \sim 8 \mathrm{~nm}$ (tubulin dimer, $\mathrm{MHz}$ ), $\lambda_{p} \sim 200 \mathrm{~nm}$ (lattice period, $\mathrm{kHz}$ ), $\lambda_{p} \sim \lambda$ (water channel, GHz), and $200 \mathrm{~nm}<\lambda<25 \mu \mathrm{m}$, hence $0.001 \Omega<R<1 \Omega(\mathrm{MHz})$ or $0.04 \Omega<R$ $<720 \Omega(\mathrm{kHz})$ as a function of length and for GHz, its $720 \Omega$ (constant). Antenna efficiency was $\leq 0.01$, which demands to bundle of microtubules like carbon nanotubes [30] for long-range wireless communication systems. DC conductivity decreases under electromagnetic measurements were first reported by Sanabria et al. [31]. However, Sanabria et al. [31] measured data only within the domain of $1 \mathrm{MHz}$.Sahu et al. [18] confirmed that and going further from $1 \mathrm{MHz}$ up to $20 \mathrm{GHz}$, showed that at certain frequencies there are sharp increments in the DC conductance. Microtubules are normally incredibly good insulators, i.e., resistance was greater than $400 \mathrm{G} \Omega$. However, the resistance decreases to a few $\mathrm{M} \Omega$; at certain frequencies, input signal reflects, as if there was a negative resistance (Ghosh et al. [7]). Looking beyond AC triggered plasmonic, for a polaronic case, the microtubule's length is the wavelength of IR radiation i.e., $\lambda \sim 25 \mu \mathrm{m} P \sim(\lambda \mathrm{n})^{2}, \mathrm{n}=$ integer). Oscillatory conductance jump with temperature was identified as IR emission. The cavities for the $\mathrm{kHz}, \mathrm{MHz}$ and $\mathrm{GHz}$ antenna-receiver actions are C-termini/MAP, secondary structures of tubulin and water channel respectively, it was not 
a classical dipolar antenna [32] origin of resonance is the presence of multiple conduction pathways along the microtubule [8]. Multiple resonance peaks at a wide range of frequencies are required to match impedances for the high-resolution communication in a complex network of antennas. In microtubule, $\mathrm{kHz}$ and $\mathrm{GHz}$ band-based communications offer robust impedance match due to wide bandwidth $(\mathrm{Q} \sim 2)$, which nullifies the antenna's directivity. The closely spaced yet discrete MHz band ensures secured phase-locked communication (narrowband $Q \sim 300$ ). In MHz domain, microtubule can create a phased array of the current source, by changing its length (period controls $\lambda_{p}$ ) and received frequency. Its $\sim 300 \mathrm{M} \Omega$ contact resistance suggests that antenna-receiver protocol offers the most feasible and reliable communication mode.

\subsection{Triplet of Triplet Fractal Ionic Resonance Band of a Single Neuron}

As above, we mapped the frequency response of a single microtubule. One basic problem of developing a similar frequency map of a neuron is to find a trick to fine-tune the sub-threshold neural firing. We carried out an extensive search to find a set of frequencies using coaxial probe and patch-clamp simultaneously as shown in Figure 1d,e targeting the axon initial segment (AIS) so that while operating at $5-20 \mathrm{mV}$ below the threshold of $-55 \mathrm{mV}$, i.e., at around $-65 \mathrm{mV}$ to $-60 \mathrm{mV}$, we control the transmission along the nerve membrane. We artificially send a wireless AC signal at resonance frequencies of microtubule $(220 \mathrm{MHz})$ using an antenna locating it very near to the AIS and monitor using our coaxial atom probe what neuron does after absorbing the signal. Two coaxial probes show that a signal back propagates from AIS to the soma, just opposite to the commonly believed direction of a nerve impulse. It was a decaying signal. This back-propagated signal builds up the potential of the soma. At certain frequencies $(30 \mathrm{MHz}+220 \mathrm{MHz}+7 \mathrm{GHz})$, depending on the topology of a neuron, the soma assists AIS, which eventually sends back a neural spike through axon, else, if the buildup potential was more than $-60 \mathrm{mV}$ at Soma, a full-scale firing of nerve impulse is triggered by AIS. Vertical gates make sure that neuron does not build-up to the axon potential, protein and its complexes do not resonate robustly.

We cultured a pair of rat hippocampal neurons on a pre-grown electrode array (see Experimental sections, Figure 3a). The growth was monitored so that the neural branches did not touch any electrode, using atom probes and DC fields one can regulate branching. Prior to any resonance measurement [14], we placed a pair of patch-clamp probes, rupturing the soma membrane in one/two neighboring neurons to measure the potential difference between them. We also measured the potential of a nerve impulse concerning the solution. On the chip, the AC electromagnetic signals of wide ranges of frequencies were applied via a pair of electrodes (PQ and RS Figure 3a) along the axon horizontally, as if dendritic branches are the sources of a signal and axonal branches are the drains. Perpendicular to it using MN electrode (Figure 3a) gating signal was applied to regulate the nerve impulse. PQ and RS electrodes change frequencies synchronously. Keeping the perpendicularly applied AC signal of MN fixed at a particular frequency we changed the frequency of the AC signal applied along the axon length via $\mathrm{PQ}$ and RS. We then changed the transverse AC signal frequency via $\mathrm{MN}$ and repeated the frequency scan via PQ and RS. This is how a 2D input frequency pattern is generated not just in a neuron, but in all the materials studied here. The basic electronic setup used to measure and filter the resonance frequency is described in detail recently. In addition to that a gating frequency was applied here, so, we get an interference pattern as an output of a nanowire.

On this 3D pattern, at particular pairs of horizontal and vertical AC frequencies, the neuron generates a potential for nerve impulse and releases ions $(<1 \%$ of threshold firing current, $\sim 50 \mathrm{nA}$; i.e., $\sim 100 \mathrm{pA}$ ), even at the sub-threshold biases of $20-30 \mathrm{mV}$. This potential makes the vertical axis; hence, we get a 3D resonance frequency map for the neuron. Figure 3a shows a 3D plot for the normalized firing potential (vertical axis) as a function of two perpendicular AC frequencies applied across the neuron. The map is unique because, the horizontal plane mapping the frequencies is the electromagnetic resonance and the vertical axis is intrinsic resonance causing the nerve impulse. In the resonance frequency pattern, three bright circles represent the situation when a neuron positively gated by MN 
electrodes while the low-intensity part shows that $\mathrm{MN}$ is arresting the nerve spikes. We could notice that three prime resonance frequency domains host three further resonance frequency domains inside making the triplet of triplet frequency band. Doublet and triplet of resonance frequencies is a common observation atomic orbital resonance of molecules, we find the occurrence of a similar kind of resonance behavior here.

The AC signal applied parallel to the axon triggers AIS only at three distinct frequency ranges, where a short pulse (pulse width $1 \mu \mathrm{s}$, total duration $1 \mathrm{~ms}$ ) from the patch-clamp at a sub-threshold bias ( 20-30 mV) activates the firing. An additional vertical AC signal resolves each of those three frequency domains into three additional sets; we get nine bands. Inserting two probes into the axon (Figure 1e) when we measured resonance bands across the AIS, we could see only three resonance bands in the linear plot. An ordered biologic structure exhibits a major longitudinal and a transverse vibration mode $[33,34]$ if the AC signal was applied in one direction, only one mode was probed. Resonating with both horizontal and transverse vibrations at different combinations of horizontal and vertical AC signals also reveals additional peaks inside the nine bands. Therefore, the relative angular orientations of the three smaller circles vary by $100-120^{\circ}$ in each of the three larger circles, but they unravel an additional dynamic feature hidden in the nine bands. A 3D resonance map of a neuron unravels three distinct time domains or periodic oscillations that regulate the nerve impulse.

As electrical nerve impulse forms at AIS, we measure a collective resonance of the AIS connected axon core. We have already reported the resonance behavior of axons with and without membrane in a single neuron. Consistency of triplet of triplet band with and without membrane prompts us to get inside a single microtubule that constitutes the major part of an axon.

The resonance behavior of a single isolated microtubule is reported [14] but not its 2D resonance pattern with gating. We dropped freshly reconstituted microtubule solution on the electrode grid (Figure 3b; see Experimental section) and an AC frequency scan was carried out similar to the neuron study. We measure the intensity of the transmitted signal along microtubule length (vertical axis of Figure $3 b$ ) as a function of two perpendicularly applied AC signals across the microtubule. Similar to the neuron, the microtubule exhibits a triplet-triplet resonance band, but it was electromagnetic, not ionic. Additional transverse field along with the horizontal AC pumping (using two perpendicular electrodes) changes the angular positions of the circles, but their relative areas remain constant. As a result, if we superimpose neuron's and microtubule's triplet-triplet bands we find shifts, but the common frequency/time regions never disappear. This suggests that the periodic oscillations of isolated microtubule and AIS are coupled.

An essential component of a single microtubule was a tubulin protein dimer. Tubulin protein solution was dropped in the gap of a four-probe electrode array (Figure 3c), then we applied a DC bias to orderly arrange the 15-20 molecules (Figure 3c). For 8-100 tubulins, trapped in the electrode array, the resonance band remained independent of the number of molecules or the electrode geometry. Similar to the microtubule and the neuron cell, we observed here triplet of triplet resonance bands (Figure $3 \mathrm{c}$ right). Normally, it was believed that electromagnetic resonance depends on the carriers, dispersion relation in a classic textbook would show how at different frequency regions, different carriers resonate. However, the classic dispersion relation presented in the textbooks, do not consider self-similar symmetry structures at all scales.

Inside the axon, initial segment microtubules form bundles and they are separated by $50 \mathrm{~nm}$, they are densely packed [35]. Microtubules are continuous and unidirectional along the axon initial segment (AIS) [36]. Since thousands of microtubules are aligned in a particular direction, the effective dipole moment of the bundle was high, dendrites have a typical composition of polarities [37]. Spatial self-organization of microtubules happens by polarity sorting [38], by which, a perfect polar system was generated [39]. In the central region of AIS, the microtubules form a nanoporous ordered crystalline structure, however, this ordered cylindrical region was covered with multi-layered ordered architectures up to the neuron membrane. The ankyrin beta spectrin square lattice was located just beneath the membrane. Therefore, the ordered signal transmission pathways link microtubule to neuron 
membranes. The connection between secondary structures of tubulin protein and the microtubule structure was also very well studied. The symmetry of tubulin's secondary structure's arrangement is reflected in its beta sheet's spiral arrangement and the symmetry of microtubule's arrangement of proteins in the hollow cylindrical structure is made of a spiral arrangement of tubulin dimers. Similarly, microtubules arranged in a helical shape in the axon initial segment, AIS, while beta-spectrin-actin square cells arrange spirally around the axon just below the membrane ( $3 \mathrm{~nm}$ below). Therefore, spiral symmetry is intact over $10^{6}$ spatial scale and change in the pitch, width and length or geometry, makes sure symmetries break at all scales in a self-similar manner.

The axon core, microtubule and tubulin have self-similar bands, with a common frequency region, a similar structural symmetry governs the resonance in all the three systems. Helical distribution of neural branches, rings of proteins in the axonal core, spirals of proteins in the microtubule, $\alpha$ helices in the proteins, are the common structures, and the resonant energy transmission in generic spiral symmetry follows a quantized behavior. Sahu et al. [26] have patented this feature of microtubule as a new class of fourth circuit element [26,40]. Hence, a spiral symmetry possibly ensures coupling of all the periodic oscillations.

For all the three systems, neuron, microtubule and tubulin, each of the nine circles in the triplet-triplet band has 6-8 small circles inside (Figure 3a-c). Since proteins are basic structures, they are pumped with the same resonating electromagnetic signal and simultaneously imaged (Figure 4a).

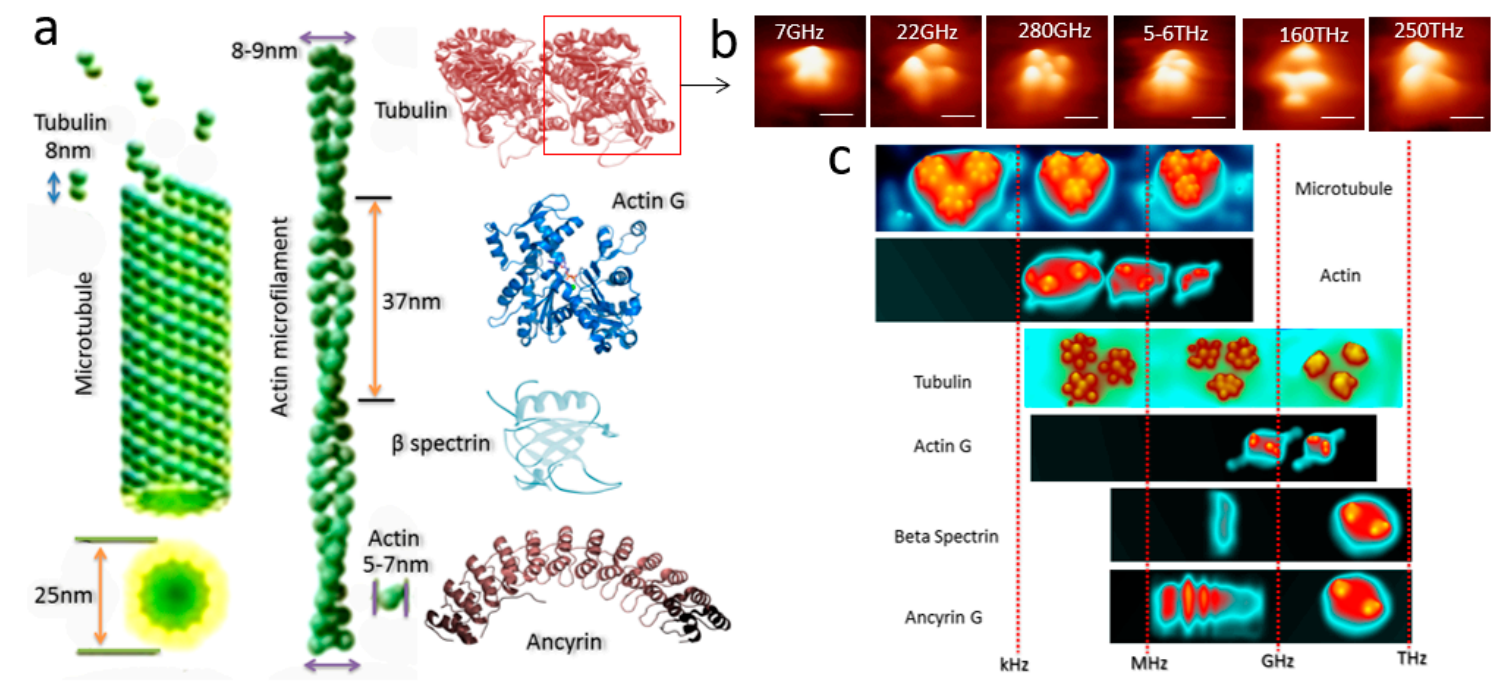

Figure 4. Generalization of 3D resonance bands for actin, $\beta$-spectrin and Ankyrin G; (a) Microtubule microfilaments, its constituent tubulin, actin protofilament and its constituent Actin G, Ankyrin and beta spectrin protein structures (pdb database); (b) Scanning Tunneling Microscope (STM) images of the monomer of a single tubulin protein, the externally applied AC frequencies at which the conformation was imaged via tunneling current is shown in the top right corner. Scale bar $=3 \mathrm{~nm}$; (c) Microtubule 3D resonance band was re-plotted from Figure 1 for comparison (top). Below, the vertical view of 3D resonance plot for actin microfilament $(800 \mathrm{kHz}-90 \mathrm{MHz}, 210-880 \mathrm{MHz}$ and 2-25 GHz), tubulin, Actin G (doublet of quartet, i.e., four circles inside a pair of circles, $232 \mathrm{GHz}-37 \mathrm{THz}$ and 60-302 THz. $\mathrm{THz}$ measurement was done by Raman spectrophotometer), beta spectrin (two pairs of circles inside a circular band 67-417 THz), Ankyrin (pair of 3, 4 peaks vary inside one circular band 23-353 THz) respectively, one in each raw. All spectra were plotted in the same frequency scale for comparison.

The resonant oscillations images of a tubulin dimer show only two high potential regions, not eight (online Movies in Ref [18]), so, dimers are not responsible for 6-8 small circles. Then as we scan the isolated tubulin monomer at various resonance frequencies, we visualize live that four major and four minor distinct potential regions inside the monomer exchange energy (Figure 4b, [18]). Total eight for a dimer. Protein dimer makes a doublet, but the monomer makes an octave. The one to one 
correspondence with the dielectric resonance image suggests that the observed 6-8 peaks in one of the 9 circles of tubulin in Figure 3 are from $\alpha$-helices localized by the $\beta$ sheets. Thus, $10^{6}$ orders of spatial journey from milli to the nanoscale execute milliseconds to sub-nanoseconds periodic oscillations. En route, the $\mathrm{GHz}$ periodic oscillation that fires a neuron originates at the single protein structure.

The triplet-triplet resonant band was not exclusive to microtubules and tubulins. We have selected mostly found four components in the axon core and around AIS, and similarly measured their temporal resonance map [41,42]. We found that actin microfilament's resonance bands are complementary to that of the microtubule, they exchange energy covering a wide frequency domain. The resonance bands for all four proteins $\beta$-spectrin, ankyrin, actin and tubulin-also their complexes are confined between the two frequency limits (Figure 4c), they share the time zone of threshold energy bursts. Overlapping time zone in the resonance frequency plot is common energy exchange regions for proteins. Thus, a resonance chain forms that connect wide ranges of proteins forming the layers beneath neuron membrane. The $\beta$-spectrin structure has ion-transfer channels and ankyrin a known mechanosensor [43] have a cascade of $\alpha$-helix oscillators dominating their resonance band. Hence, they exhibit a topological hysteresis in the 2D resonance plot. Moreover, $\beta$-spectrin and ankyrin show signatures of their lone cavities in the resonance band as doublets. A doublet in the 2D resonance pattern means the two periodic oscillations governing its resonance are coupled as part of one periodic oscillation. We understand that there are plenty of other proteins participate in generating the nerve spike. The current map is a fraction of the varieties of proteins available out there. However, the NMR-like doublets and triplets in various compositions suggest that the resonance chain would exhibit much richer topology once more proteins are added to it.

A conventional 1D resonance plot is a single line on the 2D resonance frequency map (Figure 5), represented as triplet of triplet circles (Figure 5). The triplet of triplet is not an absolute pattern-one may see pentate, or even doublets-frequency fractal or resonance chain's topology is not as simple as reported earlier [44]. The frequency wheel is created by the common time zones shared by the overlapping resonance frequencies of four proteins and their complexes studied here (Figure 5). We sonified this frequency wheel to feel how vibrating discrete time-periodic oscillations topologically integrate into a single neuron firing. In the sound file, the higher frequencies are the patterns of protein's oscillations, while the slower frequencies represent protein complexes with larger structures. A complete rotation of the wheel are events that unfold from faster to the slower time scales to eventually trigger a single nerve spike. Until now, the rapid firing of a neuron was sonified as a stream of "ticks", here we deconstruct one "tick" representing a nerve impulse with 72 frequencies bursting signals in an intricate pattern. 


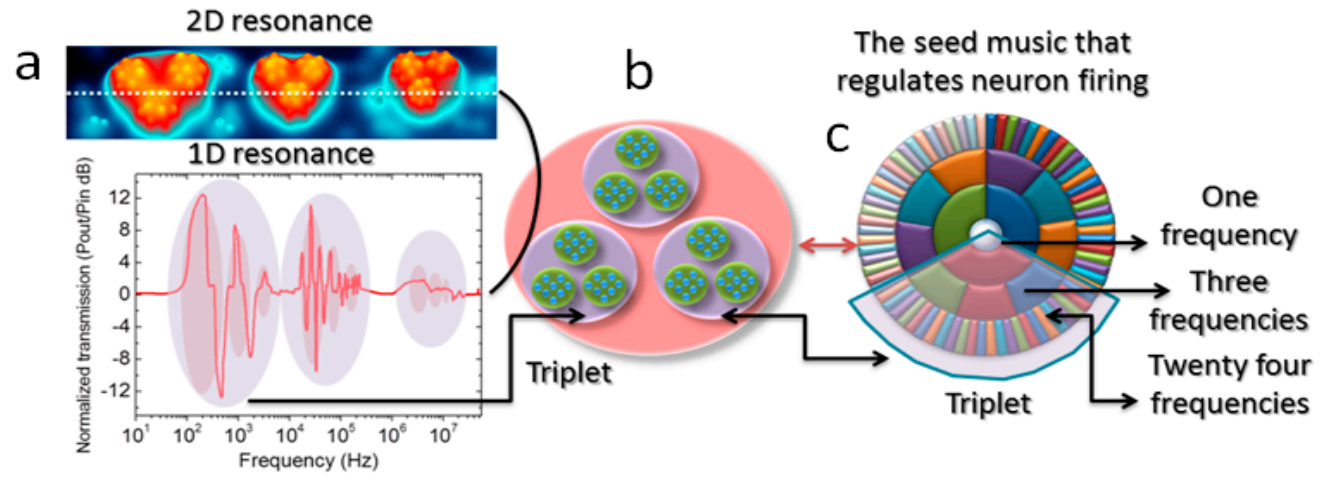

Figure 5. Frequency wheel for the triplet-triplet resonance band (Movie 1): (a) 2D resonance of a single isolated microtubule from Figure 3a. A dotted line shows 1D resonance measurement location (top), the data are shown below panel (a). The 3D resonance plot of panel a is represented as a nest of nine circles (nine circles inside three circles inside one circle $=13$ circles). Using a shadow and an arrow we connected a triplet in a 1D resonance plot of panel (a), with the schematic of panel (b); (c) The circular triplet-triplet plot of panel $b$ is a replica of experimental resonance data (panel a), however, the resonant oscillations follow a periodic condition. If we apply periodic limits then panel b looks like panel c. One can find the triplet in panel c, each triplet has single frequency and its total period is sum of three frequencies inside, each of the three has periods of eight frequencies (a particular case of tubulin).

\section{Conclusions and Future}

Dielectric resonance frequencies of multiple proteins were mapped by applying two distinct kinds of electromagnetic fields one perpendicular to another. Such an orthogonal field-based splitting of energy exchange was never applied before to read the 2D interaction of the system profile over a large frequency range. Normally 1D transmission profiles are studied, the direction at which the input signal frequency is pumped along the same direction how much the carriers are pushed through is estimated as the response of the system. When a gating effect is applied to all the three systems, additional dynamic feature hidden in the system is revealed by orthogonal fields. Such studies are regularly applied in transistor research. However, that is not the end. We speculate that if advanced further, it may be possible to map the higher-level dynamic features in the neuron, thus, we advance here the temporal correlations observed by Ghosh et al. [7] and Agrawal et al. [9]. We have detailed how a 3D triplet of triplet band could be experimentally extracted from the three systems, located one inside another $[45,46]$. The tubulins are located inside microtubule and the microtubules are located inside a neuron, if the 3D band architecture is also self-similar, it would mean that the three systems could exchange geometric information conformally, i.e., without losing the angular features of the geometric shape over a time domain of $10^{6}$ order in time scale.

Supplementary Materials: The following are available online at http://www.mdpi.com/2504-3110/4/2/11/s1, Movie 1.

Author Contributions: K.S. measured electromagnetic resonance band of microtubule using various methods; P.S. (Pushpendra Singh) did the theory of tubulin protein, microtubule and neuron using CST (Computer Simulation Technology); S.G. did all neuron measurement; S.S. measured all microtubule and tubulin protein; A.B., K.R. and P.S. (Pushpendra Singh) wrote the paper. P.S. (Pathik Sahoo) and D.F. reviewed the paper. All authors have read and agreed to the published version of the manuscript.

Funding: Authors acknowledge the Asian Office of Aerospace R and D (AOARD), a part of the United States Air Force (USAF) for Grant no. FA2386-16-1-0003 on the electromagnetic resonance-based communication and intelligence of biomaterials.

Conflicts of Interest: The authors declare no conflict of interest. 


\section{References}

1. He, B. Scale-free properties of the functional magnetic resonance imaging signal during rest and task. J. Neurosci. 2011, 31, 13786-13795. [CrossRef] [PubMed]

2. Hudetz, A.G. General Anesthesia and Human Brain Connectivity. Brain Connect. 2012, 2, 291-302. [CrossRef] [PubMed]

3. Singh, P.; Ray, K.; Fujita, D.; Bandyopadhyay, A. Complete Dielectric Resonator Model of Human Brain from MRI Data: A Journey from Connectome Neural Branching to Single Protein. In Lecture Notes in Electrical Engineering; Springer Science and Business Media LLC: Berlin/Heidelberg, Germany, 2018; Volume 478, pp. 717-733.

4. Ghosh, S.; Sahu, S.; Fujita, D.; Bandyopadhyay, A. Design and operation of a brain like computer: A new class of frequency-fractal computing using wireless communication in a supramolecular organic, inorganic systems. Information 2014, 5, 28-99. [CrossRef]

5. Singh, P.; Ocampo, M.; Lugo, J.E.; Doti, R.; Faubert, J.; Rawat, S.; Ghosh, S.; Ray, K.; Bandyopadhyay, A. Fractal and periodic biological antennas: Hidden topologies in DNA, Wasps, and retina in the eye. Stud. Comput. Intell. 2018, 761, 113-130.

6. Anguera, J.; Andújar, A.; Jayasinghe, J.; Chakravarthy, V.S.S.S.S.; Chowdary, P.S.R.; Ali, T.; Pijoan, J.L.; Cattani, C. Fractal Antennas: An Historic Perspective. Fractal Fract. 2020, 4, 3. [CrossRef]

7. Ghosh, S.; Sahu, S.; Agrawal, L.; Shiga, T.; Bandyopadhyay, A. Inventing a co-axial atomic resolution patch clamp to study a single resonating protein complex and ultra-low power communication deep inside a living neuron cell. J. Integr. Neurosci. 2016, 15, 403-433. [CrossRef]

8. Whittier, J.E.; Goddard, G.R. Microtubule Structural Dynamics Measured with Impedance Spectroscopy. FASEB J. 2006, 20, A492.

9. Agrawal, L.; Sahu, S.; Ghosh, S.; Shiga, T.; Fujita, D.; Bandyopadhyay, A. Inventing atomic resolution scanning dielectric microscopy to see a single protein complex operation live at resonance in a neuron without touching or adulterating the cell. J. Integr. Neurosci. 2016, 15, 435-462. [CrossRef]

10. Jelinek, F.; Pokorný, J. Microtubules in Biological Cells as Circular Waveguides and Resonators. Electro Magn. 2001, 20, 75-80. [CrossRef]

11. Hameroff, S.R.; Watt, R.C. Information processing in microtubules. J. Theor. Boil. 1982, 98, 549-561. [CrossRef]

12. Sahu, S.; Ghosh, S.; Hirata, K.; Fujita, D.; Bandyopadhyay, A. Multi-level memory-switching properties of a single brain microtubule. Appl. Phys. Lett. 2013, 102, 123701. [CrossRef]

13. Cosic, I.; Cosic, D.; Lazar, K. Is it possible to predict electromagnetic resonances in proteins, DNA and RNA? Nonlinear Biomed. Phys. 2015, 3. [CrossRef]

14. Sahu, S.; Ghosh, S.; Ghosh, B.; Aswani, K.; Hirata, K.; Fujita, D.; Bandyopadhyay, A. Atomic water channel controlling remarkable properties of a single brain microtubule: Correlating single protein to its supramolecular assembly. Biosens. Bioelectron. 2013, 47, 141-148. [CrossRef] [PubMed]

15. Tuszynski, J.A.; Friesen, D.; Freedman, H.; Sbitnev, V.I.; Kim, H.; Santelices, I.; Kalra, A.P.; Patel, S.D.; Shankar, K.; Chua, L.O. Microtubules as Sub-Cellular Memristors. Sci. Rep. 2020, 10, 2108. [CrossRef]

16. Kalra, A.P.; Patel, S.D.; Bhuiyan, A.F.; Preto, J.; Scheuer, K.G.; Mohammed, U.; Lewis, J.D.; Rezania, V.; Shankar, K.; Tuszynski, J.A. Investigation of the Electrical Properties of Microtubule Ensembles under Cell-Like Conditions. Nanomaterials 2020, 10, 265. [CrossRef]

17. Priel, A.; Ramos, A.J.; Tuszynski, J.A.; Cantiello, H.F. A Biopolymer Transistor: Electrical Amplification by Microtubules. Biophys. J. 2006, 90, 4639-4643. [CrossRef]

18. Sahu, S.; Ghosh, S.; Fujita, D.; Bandyopadhyay, A. Live visualizations of single isolated tubulin protein self-assembly via tunneling current: Effect of electromagnetic pumping during spontaneous growth of microtubule. Sci. Rep. 2014, 4, 7303. [CrossRef]

19. Minoura, I.; Muto, E. Dielectric Measurement of Individual Microtubules Using the Electroorientation Method. Biophys. J. 2006, 90, 3739-3748. [CrossRef]

20. Umnov, M.; Palusinski, O.A.; Deymier, P.A.; Guzman, R.; Hoying, J.; Barnaby, H.; Yang, Y.; Raghavan, S. Experimental evaluation of electrical conductivity of microtubules. J. Mater. Sci. 2006, 42, 373-378. [CrossRef]

21. Langmuir, I. The Role of Attractive and Repulsive Forces in the Formation of Tactoids, Thixotropic Gels, Protein Crystals and Coacervates. J. Chem. Phys. 1938, 6, 873. [CrossRef] 
22. Ebbinghaus, S.; Kim, S.J.; Heyden, M.; Yu, X.; Heugen, U.; Gruebele, M.; Leitner, D.M.; Havenith, M. An extended dynamical hydration shell around proteins. Proc. Natl. Acad. Sci. USA 2007, 104, 20749-20752. [CrossRef] [PubMed]

23. Mershin, A.; Kolomenski, A.; Schuessler, H.; Nanopoulos, D. Tubulin dipole moment, dielectric constant and quantum behavior: Computer simulations, experimental results and suggestions. Biosystems 2004, 77, 73-85. [CrossRef] [PubMed]

24. Otting, G.; Liepinsh, E.; Wüthrich, K. Protein hydration in aqueous solution. Science 1991, 254, 974-980. [CrossRef] [PubMed]

25. Denisov, V.P.; Peters, J.; Hörlein, H.D.; Halle, B. Using buried water molecules to explore the energy landscape of proteins. Nat. Struct. Mol. Boil. 1996, 3, 505-509. [CrossRef]

26. Satyajit, S.; Daisuke, F.; Anirban, B. Helical symmetries observed in proteins: An inductor made of arrayed capacitors (2010) Japanese patent has been issued on 20th August 2015 JP-511630 (world patent filed, this is the invention of fourth circuit element). U.S. Patent 9019685B2, 28 April 2015.

27. Borisy, G.G.; Marcum, J.M.; Olmsted, J.B.; Murphy, D.B.; Johnson, K.A. Purification of Tubulin and Associated High Molecular Weight Proteins from Porcine Brain and Characterization of Microtubule Assembly in Vitro. Ann. N. Y. Acad. Sci. 1975, 253, 107-132. [CrossRef]

28. Fygenson, D.K.; Braun, E.; Libchaber, A. Phase diagram of microtubules. Phys. Rev. E 1994, 50, 1579-1588. [CrossRef]

29. Pizzi, R.; Strini, G.; Fiorentini, S.; Pappalardo, V.; Pregnolatio, M. Evidences of new biophysical properties of microtubule. In Artificial Neural Networks; Kwon, S.J., Ed.; Nova Science Publications: New York, NY, USA, 2010; pp. 1-17.

30. Attiya, A. Lower Frequency Limit of Carbon Nanotube Antenna. Prog. Electromagn. Res. 2009, 94, 419-433. [CrossRef]

31. Sanabria, H.; Miller, J.H., Jr.; Mershin, A. Impedance spectroscopy of alpha-beta tubulin heterodimer suspensions. Biophys. J. 2006, 90, 4644-4650. [CrossRef]

32. Hanson, G. Fundamental transmitting properties of carbon nanotube antennas. IEEE Trans. Antennas Propag. 2005, 53, 3426-3435. [CrossRef]

33. Pokorny, J.; Jelenek, F.; Trkval, V.; Lamprecht, I.; Holtzel, R. Vibrations in microtubules. J. Biol. Phys. 1997, 48, 261-266.

34. Daneshmand, F.; Amabili, M. Coupled oscillations of a protein microtubule immersed in cytoplasm: An orthotropic elastic shell modeling. J. Boil. Phys. 2012, 38, 429-448. [CrossRef] [PubMed]

35. Hirokawa, N. Cross-linker system between neurofilaments, microtubules and membranous organelles in frog axons revealed by the quick-freeze, deep-etching method. J. Cell Boil. 1982, 94, 129-142. [CrossRef] [PubMed]

36. Heidemann, S.R.; Landers, J.M.; Hamborg, M.A. Polarity orientation of axonal microtubules. J. Cell Boil. 1981, 91, 661-665. [CrossRef] [PubMed]

37. Baas, P.W.; Deitch, J.S.; Black, M.M.; Banker, G.A. Polarity orientation of microtubules in hippocampal neurons: Uniformity in the axon and nonuniformity in the dendrite. Proc. Natl. Acad. Sci. USA 1988, 85, 8335-8339. [CrossRef]

38. Heidemann, S.R.; Hamborg, M.A.; Thomas, S.J.; Song, B.; Lindley, S.; Chu, D. Spatial organization of axonal microtubules. J. Cell Boil. 1984, 99, 1289-1295. [CrossRef]

39. Craig, E.M.; Yeung, H.T.; Rao, A.; Baas, P.W. Polarity sorting of axonal microtubules: A computational study. Mol. Boil. Cell 2017, 28, 3271-3285. [CrossRef]

40. Sahu, S.; Fujita, D.; Bandyopadhyay, A. Helical Symmetries Observed in Proteins: An Inductor Made of Arrayed Capacitors. Japanese Patent JP-511630, 20 August 2015.

41. Leterrier, C.; Vacher, H.; Fache, M.P.; d'Ortoli, S.A.; Castets, F.; Autillo-Touati, A.; Dargent, B. End-binding protiens EB3 and EB1 link microtubules to ankyrin $\mathrm{G}$ in the axon initial segment. Proc. Natl. Acad. Sci. USA 2011, 108, 8826-8831. [CrossRef]

42. Xu, K.; Zhong, G.; Zhuang, X. Actin, Spectrin, and Associated Proteins Form a Periodic Cytoskeletal Structure in Axons. Science 2012, 339, 452-456. [CrossRef]

43. Lee, G.; Abdi, K.; Jiang, Y.; Michaely, P.; Bennett, V.; Marszalek, P.E. Nanospring behavior of ankyrin repeats. Nature 2006, 440, 246-249. [CrossRef] 
44. Buzsaki, G.; Moser, E.I. Memory, navigation and theta rhythm in the hippocampal-entorhinal system. Nat. Neurosci. 2013, 16, 130-138. [CrossRef]

45. Reddy, S.; Sonker, D.; Singh, P.; Saxena, K.; Singh, S.; Chhajed, R.; Tiwari, S.; Karthik, K.V.; Ghosh, S.; Ray, K.; et al. A Brain-like Computer Made of Time Crystal: Could a Metric of Prime Alone Replace a User and Alleviate Programming Forever? Stud. Comput. Intell. 2018, 761, 1-43.

46. Bandyopadhyay, A. Nanobrain: The Making of an Artificial Brain from a Time Crystal, 1st ed.; CRC Press: Boca Raton, FL, USA, 2020. [CrossRef]

(C) 2020 by the authors. Licensee MDPI, Basel, Switzerland. This article is an open access article distributed under the terms and conditions of the Creative Commons Attribution (CC BY) license (http://creativecommons.org/licenses/by/4.0/). 